\title{
Silent meningioma - a rare cause of stroke in post-cardiopulmonary bypass patients
}

Hon K. Chow ${ }^{1}$, Sajjad M. Yousafzai ${ }^{1,2}$, Murat Ugurlucan², Charles C. Canver ${ }^{2}$

1Division of Cardiothoracic Surgery, Department of Surgery, Hospital University Kebangsaan Malaysia, Kuala Lumpur, Malaysia

${ }^{2}$ King Faisal Heart Institute, King Faisal Specialist Hospital and Research Centre, Riyadh, Saudi Arabia

Submitted: 8 March 2009

Accepted: 5 April 2009

Arch Med Sci 2010; 6, 2: 276-280

DOI: 10.5114/aoms.2010.13908

Copyright @ 2010 Termedia \& Banach
Corresponding author:

Dr. Murat Ugurlucan

Atasehir, 35. Ada

Zumrut Sitesi

Blok: 9, Daire: 9

Atasehir/Istanbul, Turkey

Phone: +905354316787

Fax: +902122352568

E-mail:

muratugurlucan@yahoo.com

\begin{abstract}
Stroke in cardiac patients undergoing surgery is usually attributed to the use of cardiopulmonary bypass. In this report, we present an unusual cause of cerebral dysfunction: a meningioma leading to stroke, following coronary artery bypass grafting in a 62-year-old patient. Diagnosis and treatment options of the pathology are discussed with the guidance of the literature.
\end{abstract}

Key words: stroke, brain tumour, meningioma, coronary artery bypass grafting.

\section{Introduction}

Myocardial infarction is the leading cause of death in developed and developing countries and operations to treat cardiovascular diseases are routinely and very commonly performed in the current era. Like all simple surgery, open cardiac surgery has certain risks, including death. Among these risks, neurological deterioration is one of the frequently encountered problems for various reasons $[1,2]$. On the other hand, cardiac surgery in a patient with a neurological problem is also a challenging issue and requires extensive care and precautions before, during and after the surgery [3-11].

In this report we present an uncommon cause of stroke after open heart surgery: an incidental meningioma, together with diagnosis and treatment of brain tumours in the presence of a known cardiac disease requiring surgery.

\section{Case report}

A 62-year-old woman with known history of hypertension, hypercholesterolaemia and non-insulin dependent diabetes mellitus was admitted to the hospital with the diagnosis of unstable angina pectoris. Additionally, her history revealed removal of renal calculi followed by nephrectomy, cholecystectomy, and left total knee replacement when she was 24,45 and 53 years of age, respectively; otherwise she was normal and neurologically intact. Coronary angiography showed left main system stenosis ( $45 \%$ stenosis) with triple vessel coronary artery disease (left anterior descending coronary artery [LAD] with mid portion $80 \%$ stenosis, circumflex coronary artery $[\mathrm{Cx}]$ with proximal $90 \%$ stenosis, and right 
coronary artery [RCA] with $60 \%$ stenosis proximal to the crux) and good left ventricular function (ejection fraction of 60\%).

She underwent elective quadruple coronary artery bypass grafting (CABG) with left internal mammarian artery to $L A D$ and three saphenous vein grafts to the first diagonal coronary artery, first obtuse marginal coronary artery and posterior descending branch of the RCA with standard orotracheal intubation and under routine general anaesthesia with cardiopulmonary bypass (CPB). The ascending aorta was noted to be nonthrombotic on intra-operative palpation and during proximal anastomosis of the saphenous vein grafts. Surgery was uneventful with total CPB time of 160 min and single aortic cross-clamp time of $113 \mathrm{~min}$. Proximal anastomosis of the vein grafts was performed with cross-clamping. Cardiopulmonary bypass was terminated uneventfully and she was transferred to the cardiac surgery intensive care unit and managed with morphine and midazolam in the immediate and early postoperative periods.

Early extubation failed on the first postoperative day; the patient was able to move her limbs but she was drowsy. On the second postoperative day, she opened her eyes and was able to obey commands; however, she was not able to move her left arm and lower limbs. A neurological event was considered and an urgent computerized tomography (CT) scan of the brain was performed which revealed a brain tumour, suggestive of meningioma, in the left temporoparietal region, measuring $4 \times 5 \mathrm{~cm}$ and associated with marked cerebral oedema around the tumour with little mass effect (Figure 1A-C). At the same time, blood and urine samples were collected to rule out the syndrome of inappropriate secretion of anti-diuretic hormone. However, the osmolality of the plasma was in the normal range $(282 \mathrm{mOsm} / \mathrm{kg}$; normal: 280-300 mOsm/kg of water) despite being near to the low normal. Moreover, osmolality in the spot urine was in the normal range $(284 \mathrm{mOsm} / \mathrm{kg}$; normal: 50 to $1400 \mathrm{mOsm} / \mathrm{kg}$ of water) and did not significantly change in the 24-h urine (298 $\mathrm{mOsm} / \mathrm{kg}$ ). Additionally, sodium balance was also not affected and sodium measured $138 \mathrm{mEq} / \mathrm{l}$ (normal: 135-145 mEq/l) in serum and $34 \mathrm{mEq} / \mathrm{l}$ (normal: > $20 \mathrm{mEq} / \mathrm{l}$ ) and $58 \mathrm{mEq} / \mathrm{l}$ (normal: 40-220 $\mathrm{mEq} / \mathrm{l})$ in spot urine and 24-h urine, respectively.

The patient was consulted with the neurosurgery and intravenous dexamethasone (4 mg every 6 h) was started. The response to therapy was good and she was able to be extubated on the second day of steroid therapy. She managed to ambulate with a walking frame on the $6^{\text {th }}$ postoperative day. She improved progressively and was discharged home on the $16^{\text {th }}$ postoperative day, when she was ambulating freely without support. A further
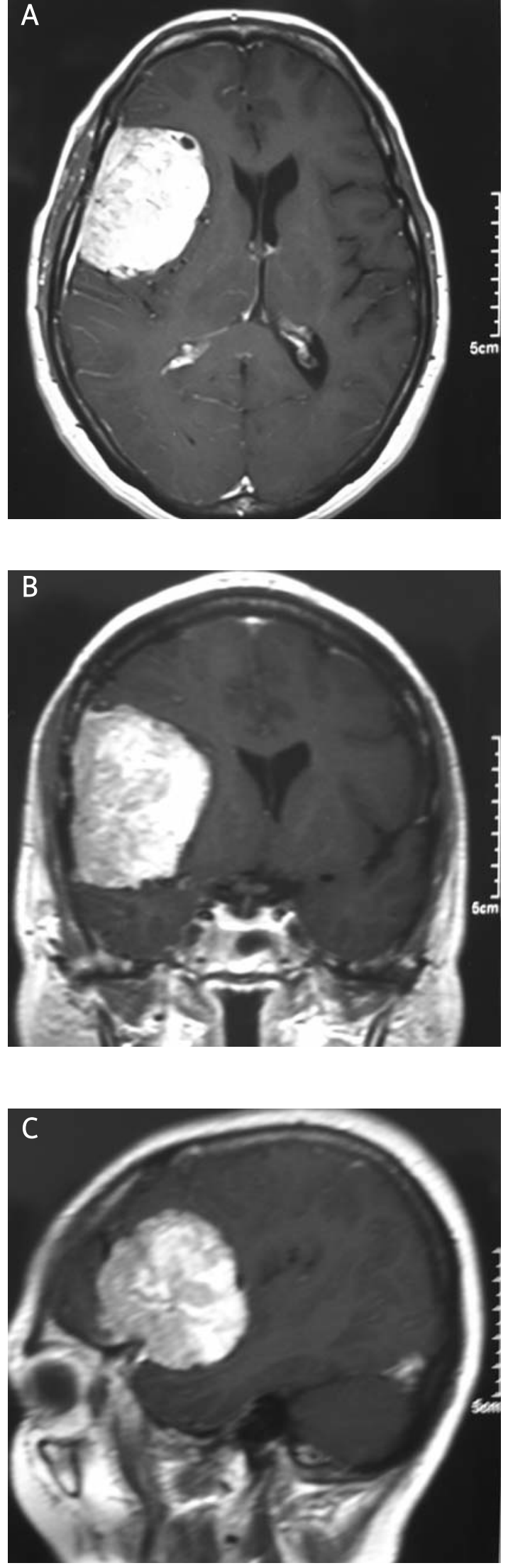

Figure 1A-C. Computerized tomography views of brain showing meningioma and peritumoural oedema at the left temporoparietal region 
appointment for neurosurgical check-up was arranged and she was scheduled for elective surgery for the treatment of meningioma.

\section{Discussion}

The incidence of meningiomas in the general population varies from 2.3 cases detected during life per 100,000 to 5.5 per 100,000 when autopsy data are included [3]. The incidence increases with age [3] and many meningiomas remain clinically silent and do not require surgery. There are only a few reports of asymptomatic brain tumours complicating the postoperative course of nonneurosurgical patients [4-6].

Predictors of stroke in patients undergoing cardiac surgery are well reported $[4,5]$ and are very helpful to identify high risk patients in whom management strategies can be altered accordingly. Preoperatively, our patient was totally asymptomatic in regards to any kind of brain tumour. Clinical presentation of meningiomas can be precipitated by anaesthesia and other perioperative factors $[5,6]$; however, although surprisingly our patient had undergone several operations under general anaesthesia, she was without any neurological problems in the postoperative periods following her previous operations.

Cardiopulmonary bypass is well known for its deleterious effects on the nervous system causing cerebral oedema as a result of altered permeability of the blood brain barrier [7]. The cause of CPBassociated alteration in blood brain barrier permeability is multifactorial but increased production of antidiuretic hormone secondary to the stress of CPB seems to play an important role in the aetiology of cerebral oedema by decreasing the plasma osmolality [8]. In our patient, laboratory tests to check serum and urine sodium and osmolality were in the normal ranges. Further diagnostic measures for the diagnosis of the syndrome of inappropriate secretion of anti-diuretic hormone, such as levels of vasopressin, atrial natriuretic and brain natriuretic peptides, renin, and aldosterone, were not performed, and the intracranial tumour became the focus of attention. Moreover, the cerebral oedema was not global and was mostly confined around the tumour, which leads to shift and hemispheric compression symptoms.

When brain tumours are encountered in patients receiving cardiac surgery, the deleterious cerebral consequences of CPB, such as impaired blood brain barrier and deteriorated cerebral autoregulation, may magnify the disturbances produced in the tumour itself [9]. In particular, meningiomas are associated with increased production of vascular endothelial growth factor, a potent angiogenic and peritumoural oedema stimulant [10]. Elevated intracranial pressure may contribute to further cerebral ischaemia and neurological dysfunction associated with cardiac surgery and CPB.

The literature includes few case reports of brain tumour patients undergoing open heart surgery. Ando et al. [11] performed aortic valve replacement successfully in the presence of a brain tumour. Similarly to our case, Kurisu et al. [12] reported delayed left hemiplegia after mitral valve replacement which was further detected to be secondary to the presence of a brain tumour with perifocal cerebral oedema. They proposed that the fluid shifts occurring within the first few postoperative days after the cardiopulmonary bypass might manifest in focal cerebral oedema and play a vital role in the clinical course of the patients [12]. Loubani et al. [13] presented their successful management for a patient with pituitary gland macroadenoma which was the cause of blindness after cardiac surgery. However, not every patient has been that lucky. The patient presented in the report by Ikeya et al. [14], who had both cardiac and cerebral angiosarcoma, died 37 days after the surgery. The CT scans showed cerebral haemorrhage on the temporal lobe with midline shift due to brain metastases [14].

Delayed recovery from anaesthesia is defined as failure to regain consciousness, responsiveness, or baseline mental status within the expected time (15-30 min) after general anaesthesia [15]. It can be due to a variety of factors (Appendix), but is most commonly caused by a relative overdosage of the anaesthetic agents $[4,6,15]$. Prolonged recovery should also raise high suspicion of a silent brain tumour after exclusion of other leading causes of delayed recovery [15]. Generally, slow recovery from anaesthesia followed by neurological deterioration is consistent with increasing intracranial pressure caused by progressive cerebral oedema. In case of delayed recovery from anaesthesia, the causes of the phenomenon, briefly summarized in the Appendix, should be recalled and patients should be carefully evaluated and managed accordingly [15].

Computed tomography or magnetic resonance imaging of the brain should be performed immediately upon suspicion of neurological insult in the early postoperative course of post-CPB patients. In our case, the CT scan of the brain confirmed the diagnosis by revealing the classical features of a solid brain tumour with severe peritumoural oedema.

Meningiomas are usually benign, slow growing tumours and may cause a slow increase in the intracranial pressure with mild or no symptoms for a long time. After displacement of blood and cerebrospinal fluid, the brain compliance decreases and a normally tolerable insult, such as general anaesthesia, may aggravate cerebral oedema [3]. 
The absence of an adverse neurological outcome after general anaesthesia in the past in our patient was probably related to the smaller tumour size and concurrent low intracranial pressure at the time of previous procedures. The reason for neurological complications other than the size of the tumour itself in our case was the insult of CPB, which seems to be more potent than the general anaesthesia alone in terms of disturbing the blood brain barrier and causing cerebral oedema.

Brain tumours cause structural damage to the cerebral endothelium, and the severity of cerebral oedema seems to be related to the size of the tumour due to cellular disruption over a wide area [7]. Deeply comatose patients should be managed with neurological and neurosurgical consultation, employing hyperventilation, cautious fluid management and anti-oedema therapy such as with steroids and mannitol. Hyponatraemia should be particularly avoided. Our patient was drowsy but arousable and had focalization in the form of left hemiplegia. The neurological deficit seemed to be related to the tumour location and size, and the severity of the peritumoural oedema. Anti-oedema therapy with intravenous dexamethasone and cautious fluid and electrolyte management are recommended in cases of focal neurological deficit with less severe deterioration of consciousness level. The role of dexamethasone in reducing peritumoural blood brain barrier permeability and inhibition of vascular endothelial growth factor action is well reported in the literature [16].

Symptomatic brain tumour patients with critical coronary artery disease and unstable angina will pose higher anaesthetic and cardiac surgical risks for postoperative stroke. This group of patients would probably benefit from off-pump CABG or a hybrid procedure using off-pump CABG with/without percutaneous interventions. Patients with brain tumours in whom CPB is unavoidable should be managed together by expert cardiac and neurosurgical anaesthesiologists with special anaesthetic management [9] starting from the preoperative period.

In conclusion, asymptomatic brain tumours should also be considered among the rare causes of stroke in patients undergoing cardiac surgery. Early neurological and/or neurosurgical consultation is strongly recommended to institute intracranial pressure management oriented therapy in time. Off-pump CABG or a hybrid procedure in patients with symptomatic brain tumour and with critical coronary artery disease may also be critically important. Other patients with known brain tumours, who are symptomatic or asymptomatic and require cardiac surgical intervention using CPB, should essentially be managed, in the perioperative period, by a team approach with the neurosurgical, cardiovascular surgery and anaesthesiology teams.

\section{References}

1. Almassi GH, Sommers T, Moritz TE, et al. Stroke in cardiac surgical patients: determinants and outcome. Ann Thorac Surg 1999; 68: 391-7.

2. Hogue CW Jr, Murphy SF, Schechtman KB, Dávila-Román VG. Risk factors for early or delayed stroke after cardiac surgery. Circulation 1999; 100: 642-7.

3. Georges Haddad, Ossama Al-Mefty. Meningiomas: an overview. In: Wilkins RH, Rengachary SS (eds). Neurosurgery. McGRAW-HILL COMPANIES (Health Professions Division). Second edition 1996; I: 833-41.

4. Giordano F, Urciuoli RM, Ragazzi P, et al. Sudden presentation of intracranial meningioma after elective general anesthesia. J Neurosurg Anesthesiol 2001; 13: 138-42.

5. Razis PA, Robinson DL, Alberry R. Clinical presentation of "silent" meningiomas after general anaesthesia. $\mathrm{Br}$ J Anaesth 1995; 74: 335-7.

6. Young HA, Smith H, Gregory SA, Gleave J. Intracranial meningioma unmasked by elective general anesthesia: report of three cases. Neurosurgery 1984; 14: 328-31.

7. Harris DN, Oatridge A, Dob D, Smith PL, Taylor KM, Bydder GM. Cerebral swelling after normothermic cardiopulmonary bypass. Anesthesiology 1998; 88: 340-5.

8. Philbin DM, Coggins CH, Wilson N, Sokoloski J. Antidiuretic hormone levels during cardiopulmonary bypass. J Thorac Cardiovasc Surg 1977; 73: 145-8.

9. Grigore AM, Grocott HP, Newman MF. Anesthetic management of a patient with a brain tumor for cardiac surgery. J Cardiothorac Vasc Anesth 2000; 14: 702-4.

10. Provias J, Claffey K, delAguila L, Lau N, Feldkamp M, Guha A. Meningiomas: role of vascular endothelial growth factor/vascular permeability factor in angiogenesis and peritumoral edema. Neurosurgery 1997; 40: 1016-26.

11. Ando Y, Kurisu K, Hisahara M. Aortic valve replacement in a patient with a brain tumor. Gen Thorac Cardiovasc Surg 2008; 56: 295-7.

12. Kurisu K, Hisahara M, Ando Y, Tominaga R. An unusual manifestation of brain tumor: development of delayed hemiplegia after cardiopulmonary bypass. J Card Surg 2007; 22: 417-8.

13. Loubani M, Galiñanes M. Pituitary gland macroadenoma: a cause of transient blindness after cardiac surgery. Ann Thorac Surg 2001; 72: 929-31.

14. Ikeya E, Taguchi J, Yamaguchi M, Shibuya M, Kanabuchi K. Primary cardiac angiosarcoma: presenting with cardiac tamponade followed by cerebral hemorrhage with brain metastases. Jpn J Thorac Cardiovasc Surg 2006; 54: 528-31.

15. Radhakrishnan J, Jesudasan S, Jacob R. Delayed awakening or emergence from anaesthesia. Update in Anesthesia 2001; 13: 1-2.

16. Heiss JD, Papavassiliou E, Merrill MJ, et al. Mechanism of dexamethasone suppression of brain tumor-associated vascular permeability in rats. Involvement of the glucocorticoid receptor and vascular permeability factor. J Clin Invest 1996; 98: 1400-8. 


\section{Appendix [15]}

\section{Causes of delayed awakening}

\section{Residual drug effects:}

- overdose,

- duration and type of anaesthetic given,

- potentiation by other drugs,

- prolonged neuromuscular blockade,

- respiratory failure.

\section{Metabolic derangements:}

- hypoglycaemia,

- severe hyperglycaemia,

- electrolyte and acid-base imbalance,

- hypothermia,

- central anticholinergic syndrome,

- sepsis,

- pre-existing encephalopathy,

- hypothermia (< 33 degrees).

\section{Neurological complications:}

- cerebral hypoxia or decreased cerebral perfusion,

- intracerebral event or stroke.

Typical situations:

- after shorter than expected cases,

- patients with renal or hepatic dysfunction,

- neonates or elderly,

- following neurological, carotid, cardiac, or vascular surgery,

- following trauma or massive fluid resuscitation. 